\title{
Discharge of repulsive grains from a silo: experiments and simulations
}

\author{
David Hernández-Enríquez ${ }^{1}$, Geoffroy Lumay ${ }^{2}$, and Felipe Pacheco-Vázquez ${ }^{1, \star}$ \\ ${ }^{1}$ Instituto de Física, Benemérita Universidad Autónoma de Puebla, Apartado Postal J-48, Puebla 72570, Mexico. \\ ${ }^{2}$ GRASP, Université de Liège, B-4000 Liège, Belgium.
}

\begin{abstract}
In granular matter, sliding friction and collisions among grains are fundamental mechanisms of energy dissipation that determine the particles dynamics. Here we consider an unconventional granular system composed of magnetic repelling grains confined in a two dimensional cell that interact only through their magnetic field. The repulsive interaction prevents contact among grains and therefore produces a different dynamics compared to the dynamics of classical granular systems. In particular, we present experiments and simulations on the discharge of this repulsive granular medium from a silo. The results reveal an inverted density profile and a plug-flow through the aperture that contrast with the dynamics displayed by contacting grains. Moreover, the simulations allow to estimate the friction coefficient generated by the lateral confinement.
\end{abstract}

\section{Introduction}

Because of its omnipresence in industry and agriculture, one of the most widely studied systems in granular matter is the discharge of grains from a silo [1-10]. In the pioneering work of Beverloo [2] describing the mass rate $Q$ of grains of size $d$ flowing through an orifice of diameter $D$ under the influence of gravity $g$, it was found that $Q=C \rho_{b} \sqrt{g}(D-\kappa d)^{5 / 2}$, where $\rho_{b}$ is the apparent density of the material and $C$ and $\kappa$ are constants related to the particle nature and silo geometry. The $5 / 2$ power dependence has been verified for different kind of particles, independently of their packing fraction, density, surface properties or shape [3]. For small apertures, where clogging is possible $(D / d \sim 10)$, an exponential corrective factor must be introduce to predict the flow due to a lower density of material near the exit [4]. Concerning the discharge of a two dimensional silo, an expression for the flow rate is given by $Q=\left\langle\rho_{b}\right\rangle \sqrt{2 g \beta D}(D-k d)$ with $\beta \sim 0.5$. One of the most relevant aspects of these expressions is the independence of $Q$ on the column height, in contrast to the hydrostatic dependence at the bottom of a water column. Recently, it has been also investigated the granular discharge of submerged hoppers, and a surge in the flow rate was measured at the end of the process[5].

A granular material is usually defined as a conglomerate of macroscopic particles characterized by a loose of energy due to elasticity and friction when particles collide or slide [6]. In a recent work [7], the author of this manuscript and colleagues presented experiments in a two dimensional cell filled with an assembly of magnets that do not interact through contact forces but through their repelling magnetic field. These contact-less particles are able to form stable piles with an average angle of repose,

\footnotetext{
^e-mail: fpacheco@ifuap.buap.mx
}

to flow through an aperture when it is large enough, and to form arches and produce jamming as it happens with conventional grains. Our previous experimental study was focused on determining the discharge flow rate for repelling grains in a two-dimensional silo. In contrast to classical granular flows, the results showed a plug flow velocity profile along the silo aperture. It was also found that the average density of grains $\langle\rho\rangle_{x, t}$ above the aperture decreases with $D$ (another difference with classical granular materials) and it was well fitted by:

$$
\langle\rho\rangle_{x, t}=\rho_{\infty}+\left(\rho_{0}-\rho_{\infty}\right) e^{-D / \alpha}
$$

where $\rho_{0}$ and $\rho_{\infty}$ are the density of grains for the static column previous to discharge and the density when the bed is in free fall, respectively, and $\alpha$ is a constant related to the minimum value of $D$ for a continuous flow. Then, the flow rate is given by:

$$
Q=\left[\rho_{\infty}+\left(\rho_{0}-\rho_{\infty}\right) e^{-D / \alpha}\right] \sqrt{2 g \beta} D^{3 / 2}
$$

This expression is independent of the column height, and therefore, Q was found to be constant as in the case of a discharge of contacting grains.

In the present paper, we show numerical results obtained from molecular dynamics(MD) simulations which are compared with the above experimental findings. These simulations allow us to better understand the effect of friction between the grains and the walls and to avoid finite size effects by keeping the grains number constant.

\section{Numerical simulations}

In our experiments only 565 magnets fit into the $1.8 \times 0.5$ $\mathrm{m}^{2}$ compartment due to the grain-grain and grain-wall repulsive interactions. The small number of particles produced finite size effects so that the average density and 

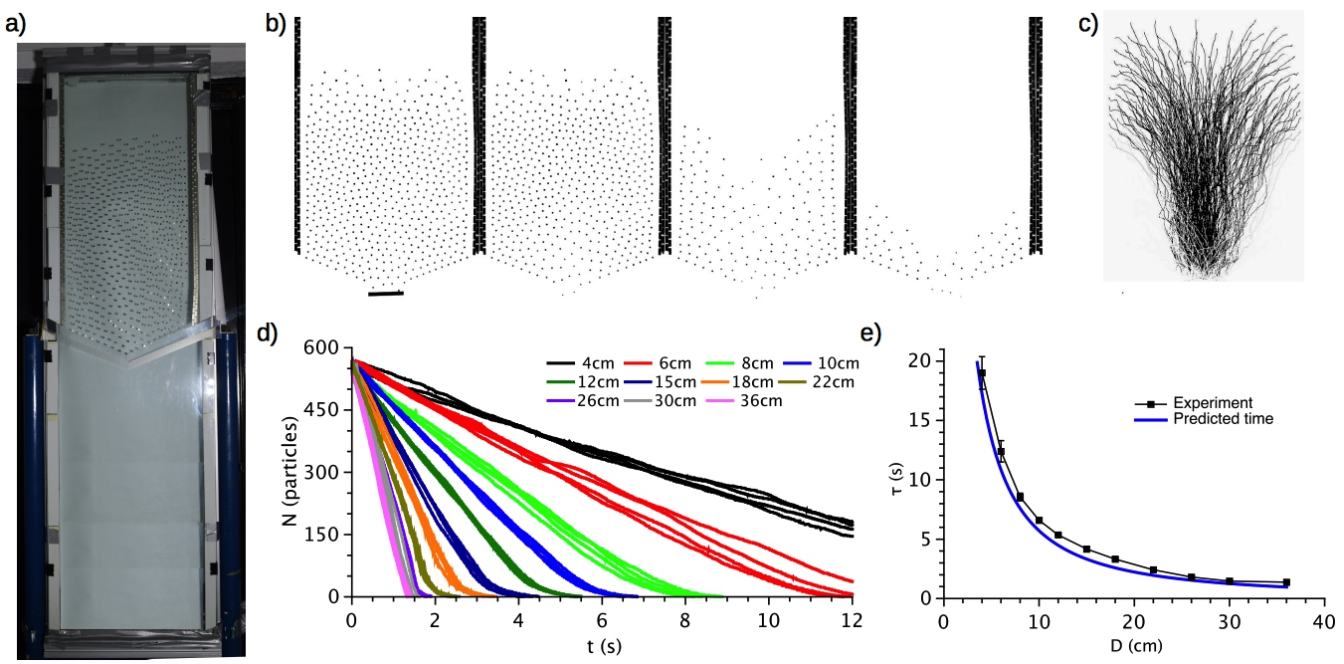

Figure 1. a) Experimental setup. b-c) Snapshots and grain paths for a silo discharge of aperture $D=8 \mathrm{~cm}$. d) Number of particles $N$ remaining in the upper compartment as a function of time $t$ for different values of $D$. e) Total time of discharge $\tau$ vs $D$.

velocities were computed in a reduced time interval of the whole discharge. Since building a larger cell was unworkable, and aiming to validate the experimental results, we performed MD simulations considering disks of diameter $\mathrm{d}=5 \mathrm{~mm}$ with a repulsive potential in twodimensions. The non-linear repulsive force $\vec{F}(r, \theta)$ between two magnetic dipoles separated by a distance $r$ with an angle $\theta$ between the direction of the magnetic field and the line joining the dipoles centres is given by: $\vec{F}(r, \theta)=\left(3 \mu m^{2} / 4 \pi r^{4}\right)\left[\left(1-3 \cos ^{2} \theta\right) \hat{r}+\sin 2 \theta \hat{\theta}\right]$, where $\mu_{0}$ is the vacuum magnetic permittivity and $m$ is the dipolar magnetic moment. The total repulsive force over each magnet $\vec{F}_{i}\left(x_{i}(t)\right)$ was calculated considering its interaction with the rest of the disks at distances $r_{i j}$. In the twodimensional configuration, $\theta=\pi / 2$, and therefore the force over the $\mathrm{i}$-th magnet at a given time $t$ is given by:

$$
\vec{F}_{i}\left(x_{i}(t)\right)=\sum_{j \neq i}^{n} \frac{3 \mu_{0} m^{2}}{4 \pi r_{i j}^{4}} \widehat{r}_{i j}
$$

This expression was introduced in the velocity Verlet algorithm[11] programmed in Matlab ${ }^{\circledR}$ to integrate the equations of motion, where positions $x_{i}$, velocities $v_{i}$ and accelerations $a_{i}$ at time $t+\Delta t$ are obtained from the same quantities at time $\mathrm{t}$ according to:

$$
\begin{gathered}
\overrightarrow{x_{i}}(t+\Delta t)=\overrightarrow{x_{i}}(t)+\overrightarrow{v_{i}}(t) \Delta t+\frac{1}{2} \overrightarrow{a_{i}}(t) \Delta t^{2} \\
\overrightarrow{v_{i}}(t+\Delta t)=\overrightarrow{v_{i}}(t)+\frac{\overrightarrow{a_{i}}(t)+\overrightarrow{a_{i}}(t+\Delta t)}{2} \Delta t \\
\overrightarrow{a_{i}}(t+\Delta t)=\frac{\vec{F}_{i}\left(x_{i}(t)\right)+\vec{\xi}_{i}}{m_{i}}
\end{gathered}
$$

In eq.(6), the term $\vec{\xi}_{i}$ represents a dissipative force associated to friction due to the glass walls.

The simulation begins with 565 disks in a rectangular array with random velocities taken from a Gaussian distribution. The discs are dropped under gravity into a silo of appropriate dimensions and geometry according to the experiments. Once all the grains have dropped and the change in the average kinetic energy between two consecutive time steps is less than a certain value $\epsilon$, the gate is open to start the discharge and the flow is measured by counting the number of particles that have fallen for each time step $\Delta t$. In order to increase the total discharge time and neglect finite size effects, the number of grains $N$ was kept constant during the simulation by reinserting the outgoing grains in the upper part of the silo. The simulation ends when 565 magnets have passed through the aperture. The simulations were performed with and without the dissipative term to determine the effect of the confinement.

\section{Results}

Let us first describe the experimental set-up and some results that were necessary to calibrate our simulations. Figure 1a shows the cell filled with $N_{\text {tot }}=565$ cylindrical Neodymium magnets oriented with their magnetic dipoles pointing in the same direction to induce a repulsive force among them; the lateral walls were also repulsive and the aperture size $D$ was set prior to discharge. Because there is no contact among grains, they can be easily distinguished and tracked. Figure $1 \mathrm{~b}$ shows snapshots of the discharge for a small aperture $D=8 \mathrm{~cm}$; the paths followed by the grains in fig. 1c indicate a funnel flow pattern as it occurs with non-repelling grains. From videos taken at $250 \mathrm{fps}$, we counted the number of particles $N$ remaining at the upper cell compartment as a function of time $t$ for different values of $D$ ranging from $4 \mathrm{~cm}$ to $36 \mathrm{~cm}$; the results are shown in fig. 1d. There are two different behaviours: a linear decrease during most of the discharge that reflects a constant flow rate $Q$ (constant slope) and a decrease of the flow at the end of the process. The total discharge time $\tau$ is plotted as a function of $D$ in fig. 1e for the experiments (points) and compared with the expected value $\tau=N_{\text {tot }} / Q$ (blue line). 
a)

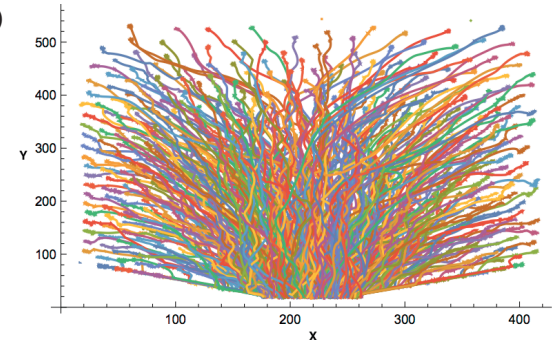

b)

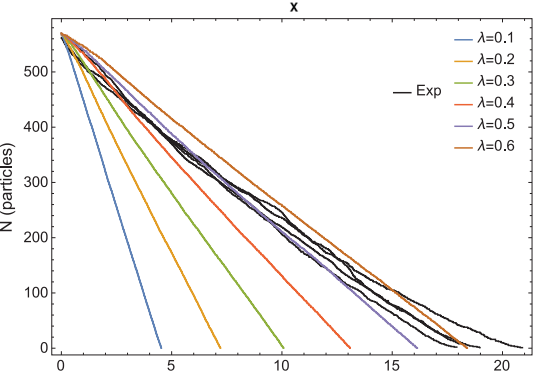

$t(s)$

c)

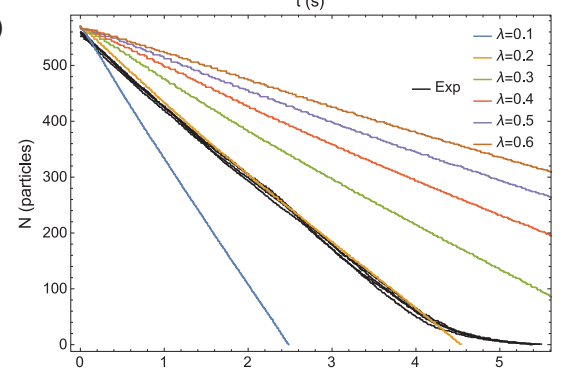

d)

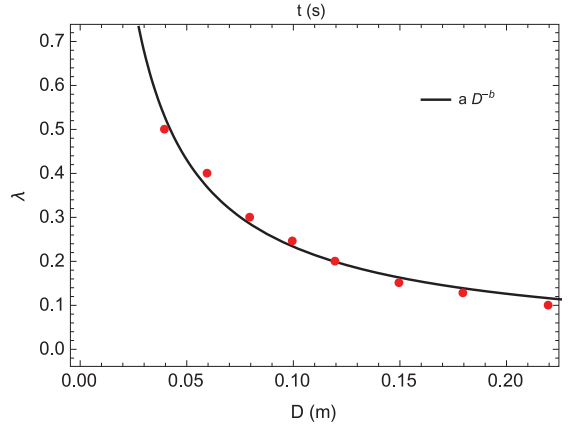

Figure 2. MD simulations: a) Particles paths for $D=8 \mathrm{~cm}, \mathrm{~b}-\mathrm{c}$ ) $N$ vs $t$ for $D=4 \mathrm{~cm}$ and $12 \mathrm{~cm}$, respectively, for different values of $\lambda$. Black lines represent the experiments. d) $\lambda$ vs $D$ obtained from the best comparison with experimental results (red points); the black line corresponds to $\lambda=(0.03 \pm 0.002) D^{-0.89 \pm 0.07}$.

Figure 2a shows the trajectories of 565 repulsive particles obtained from the simulations. Note that, as in the experimental case, the magnets move mainly along the central part of the cell developing a funnel flow. We performed simulations with different dependences for $\vec{\xi}_{i}$ but only a viscous term of the form $\vec{\xi}_{i}=\lambda \overrightarrow{v_{i}}$ was able to reproduce the linear behaviour of $N$ vs $t$ found in the experiments. The friction coefficient $\lambda$ was varied until obtaining the best comparison with the experimental data for each value of $D$, see figs. 2b-c. Figure $2 \mathrm{~d}$ shows that $\lambda$ decreases with $D$ and it is well fitted by $\lambda=(0.03 \pm 0.002) D^{-0.89 \pm 0.07}$. To explain this dependence, let us consider that the torque $\vec{\tau}$ on a magnetic dipole $\vec{\mu}$ placed in a magnetic field $\vec{B}$ is directly related to the strength of the field by $\vec{\tau}=\vec{\mu} \times \vec{B}$; if the dipole is free a)

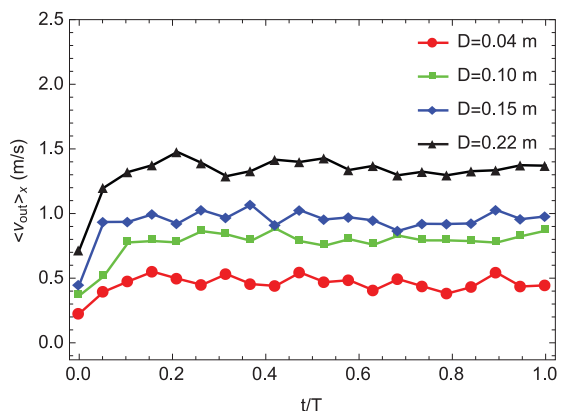

b)

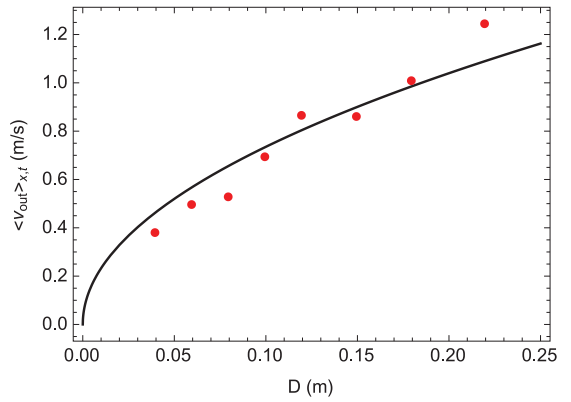

c)

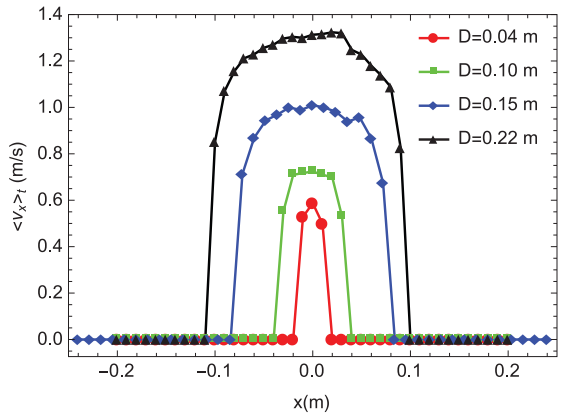

Figure 3. a) $\left\langle v_{\text {out }}\right\rangle_{x}$ vs $t$ for four different apertures; $t$ is normalized by the discharging time $T$ of 565 magnets. b) $\left\langle v_{\text {out }}\right\rangle_{x, t}$ vs $D$. c) Velocity profile along the aperture.

to move, it will rotate until $\vec{\mu}$ is parallel to $\vec{B}$. In the cell, the frontal walls prevent the magnet rotation but the torque over each magnet produces an increase of friction with the walls which is proportional to $\vec{\tau}$. In the experiments, the separation among particles is observed to increase with $D$, reducing the local density; therefore, the magnetic field over each particle $\vec{B}_{i}$ decreases and also $\vec{\tau}_{i}$, this causes the decreasing friction coefficient observed in fig. $2 \mathrm{~d}$.

Once $\lambda$ was found for each value of $D$, we computed the average particle velocity at the output $\left\langle v_{\text {out }}\right\rangle_{x}$ as a function of time; the results for four different apertures are shown in fig. 3a (notation \langle\rangle$_{x}$ indicates the average over the aperture). Note that $\left\langle v_{\text {out }}\right\rangle_{x}$ practically has a constant value during the whole discharge as it is expected for a constant flow rate. By plotting $\left\langle v_{\text {out }}\right\rangle_{x}$ averaged on time as a function of $D$ and using $\left\langle v_{\text {out }}\right\rangle_{x, t}=\sqrt{2 g \beta D}$ to fit the data, see fig. $3 \mathrm{~b}$, one finds $\beta \approx 0.27 \pm 0.05$, in contrast with $\beta \approx 0.5$ found for contacting grains in which case a free-fall semicircular arch region of size proportional to the aperture is assumed. The different value obtained with repelling grains could be associated to the fact that the free-fall region does not really exist [8], since even in the aperture the outgoing magnets are interacting with the magnetic field of the neighbouring particles. Figure $3 c$ 
a)

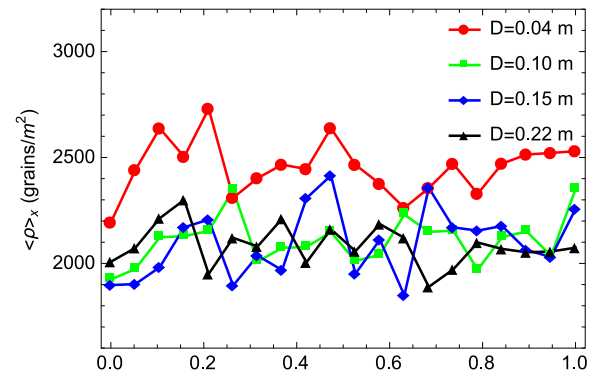

b)

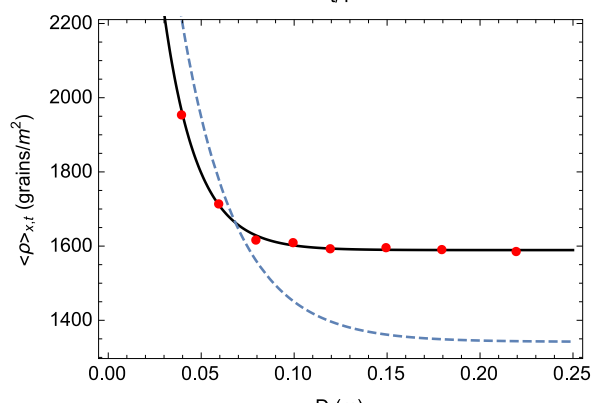

c)

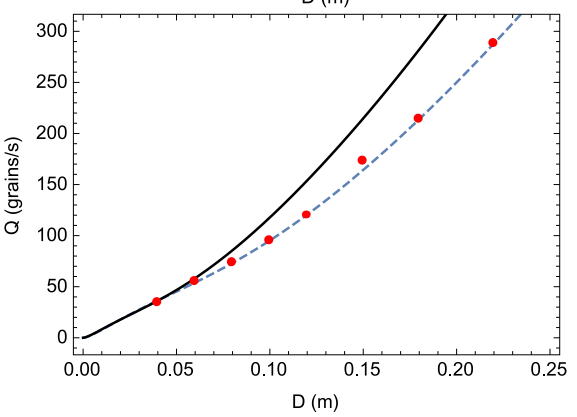

Figure 4. a) $\langle\rho\rangle_{x}$ vs $t / T$ found in simulations. b) $\langle\rho\rangle_{x, t}$ vs $D(\bullet)$, data fitting using eq.1 (-), and experimental fitting reported in [7] (- -). c) $Q=N_{\text {tot }} / \tau$ vs $D(\bullet)$ and solution of eq. 2 with parameters found in simulations (-) and those found in experiments [7] (- -).

shows the velocity profile along the aperture obtained from the simulations, $\left\langle v_{x}\right\rangle_{t}$ is the average velocity in each coordinate $x$ during the total time of discharge and $\left\langle v_{x}\right\rangle_{t}=0$ outside the aperture corresponds to the static magnets on the gates. As in the experiments, an important velocity gradient is observed near the borders of the aperture with a plug flow in the central zone; this plug flow constitutes another strong differentiation with classical granular flows.

Finally, we computed the density of particles $\langle\rho\rangle_{x}$ inside a rectangular box of $10 d \times D$ centred in the silo aperture as it was done with the experimental measurements in [7]. We found fluctuations around a mean value during the evolution of the discharge, see fig. 4a. In the experimental results, it was found a constant density only during an intermediate interval of the process due to the reduced number of grains; this effect is avoided in the simulations by reinserting the outgoing grains at the top of the silo, giving as a result a constant $\langle\rho\rangle_{x}$. The average density taken over time $\langle\rho\rangle_{x, t}$ decreases with $D$, see red points in fig. $4 \mathrm{~b}$, supporting the previous discussion related to the friction factor and in agreement with experiments. Using eq. (1) to fit the numerical results we obtain the values $\rho_{\infty}=1589.26$ grains $/ \mathrm{m}^{2}, \rho_{0}=5049.47$ grains $/ \mathrm{m}^{2}$ and $\alpha=0.0177$. These values were used to calculate $Q$ as a function of $D$ with eq.
(2); the results are compared in fig. $4 \mathrm{c}$ with the fit of the experiments reported in [7]. The difference between simulations (black line) and the experimental data fit (dashed blue line) is again associated to finite size effects.

\section{Conclusions}

We studied numerically the discharge of repelling magnetic grains from a two-dimensional silo. Our results are in agreement with previous experimental findings[7] without the limiting effects produced by the small number of grains and finite size of the system. The magnets move towards the aperture developing a funnel flow pattern as it occurs in classical granular materials, but in contrast, a plug-flow profile appears at the exit. The density of grains measured just above the silo output is found to decrease exponentially with the aperture size in accordance with the experiments. The magnetic interaction produces a torque that generates friction with the walls, the simulations allowed us to evaluate this friction factor which diminishes with $D$ as a consequence of the decreasing density. Of particular interest could be to compare our results with the dynamical scenario that typically occurs when cars pass through a bottleneck, where no surface-surface interactions also exist.

\section{Acknowledgements}

This research was supported by CONACyT Mexico through the project No. 242085 of the Sectoral Research Fund for Education, PROFOCIE-SEP 2015-2016 and VIEP-BUAP. The authors thank J. Schockmel, and S. Dorbolo for contributions and discussions. G.L thanks FNRS (Grant PDR T.0043.14) and ULG (Grant C-13/88).

\section{References}

[1] I Zuriguel, Papers in Physics 6, 060014 (2014)

[2] W. A. Beverloo, H. A. Leniger, J. van de Velde, Chem. Eng. Sci. 15, 260 (1961).

[3] D. Hirshfeld, Y. Radzyner and D. C. Rapaport, Phys. Rev. E 56, 4404-4415 (1997).

[4] C. Mankoc, et al., Granular Matter 9, 407 (2007).

[5] T. Wilson, C. Pfeifer, N. Mesyngier, and D. Durian, Pap. Phys. 6, 060009 (2014).

[6] J. Duran, Sands, Powders, and Grains: An Introduction to the Physics of Granular Materials, (SpringerVerlag, New York, 2000).

[7] G. Lumay, et al., Pap. Phys. 7, 070013 (2015).

[8] S. M. Rubio-Largo, A. Janda, D. Maza, I. Zuriguel, and R. C. Hidalgo, Phys. Rev. Lett. 114, 238002 (2015).

[9] R. O. Uñaca, A. M. Vidales and L. A. Pugnaloni, J. Stat. Mech. 2012, 04008 (2012)

[10] H. Ahn, Z. Başaranoğlu, M. Yilmaz, A. Buğutekin, and M. Zafer Gül, Powder Technol. 186, 65 (2008).

[11] Q. Spreiter and M. Walter, J. Comput. Phys. 152, 102-119 (1999). 